\title{
Aarroo Temene \\ MÉTOdo PARA DETERMINAÇÃo DE DISTRIBUIÇÃo de tAMANHo DE MICROBOLHAS (DTMB) EM SISTEMAS FLOTAÇÃo (FAD) PARA TRATAMENTO DE ÁGUAS UTILIZANDO A ANÁLISE DE IMAGEM DIGITAL
}

\section{METHOD FOR MEASURING BUBBLES SIZES DISTRIBUTION (BSD) IN DRINKING WATER TREATMENT FLOTATION (DAF) SYSTEMS BY USING DIGITAL IMAGE ANALYSIS}

\begin{abstract}
RODRIGO BRAGA MORUZZI
Professor Assistente do Departamento de Planejamento Territorial e Geoprocessamento do Instituto de Geociências e Ciências Exatas - Universidade Estadual Paulista (UNESP). Engenheiro Civil, Mestre e Doutor em Hidráulica e Saneamento

\section{Marco Antonio Penalva Reali}

Professor Doutor do Departamento de Hidráulica e Saneamento da Escola de Engenharia de São Carlos - Universidade de São Paulo. Engenheiro Civil, Mestre e Doutor em Hidráulica e Saneamento

Código ABES:124/06 Recebido: 14/09/06 Aceito: 18/06/07
\end{abstract}

\section{RESUMO}

O artigo apresenta um método proposto para determinação de tamanho de microbolhas de ar (DTMB) em sistemas de tratamento de água por flotação por ar dissolvido (FAD), utilizando a análise de imagem digital. Adicionalmente, é apresentado um estudo de caso com emprego de unidade em escala piloto. A aquisição das imagens foi realizada com uma câmera digital de 5.1MPixel, acoplada a conjunto de lentes. As imagens de microbolhas foram adquiridas em sistema com escoamento contínuo para eliminar as interferências causadas pela captura de amostras. O tratamento e obtenção das feiçôes foram realizadas com o programa (freeware) "ImageTool". Os resultados apresentaram coerência com os valores reportados na literatura utilizando métodos tradicionais. O sistema mostrou-se eficaz e o método desenvolvido pode constituir ferramenta útil para estudos em outras subáreas de conhecimento.

PALAVRAS-CHAVE: Partículas, análise de imagem, tratamento de água e flotação por ar dissolvido.

\begin{abstract}
An image analysis method proposed for assessing bubble size distribution applied to dissolved air fotation (DAF) systems for drinking water treatment is presented. Further, a pilot plant investigation is presented. A digital camera together with a group of lens was used. In order to eliminate samples interferences the images were captured in a continuous flow system working in steady state. The freeware ImageTool was applied for treating and obtaining size distribution. Results were presented in good relationship with traditional methods. The role image system showed to be efficient for image capturing and the proposed method may be an useful tool to other researches areas.
\end{abstract}

KEYWORDS: Particle size, particle size distribution, water and wastewater treatment.

\section{INTRODUÇÃO}

A flotação por ar dissolvido (FAD) com recirculação pressurizada do efluente caracteriza-se como um processo trifásico de separação sólido/líquido (Reali, 1991). Neste, microbolhas de ar são introduzidas na massa líquida visando à remoção dos flocos, formados na etapa de pré-tratamento da água.

Há mais de oito décadas a flotação por ar dissolvido (FAD) vem sendo aplicada no tratamento de águas residuárias e destinadas ao abastecimento.
No Brasil, a Estação de Tratamento de Água (ETA) de Meaípe-ES foi uma das primeiras a empregar a FAD como unidade de clarificação, inaugurada oficialmente em 1994 mas em testes desde 1990. Richter e Gross (2000) apontam também como uma das pioneiras a ETA Döhler, em Joinville-SC, inaugurada em 1992. O aumento das pesquisas e da experiência adquirida com as unidades em operação proporcionou muitos avanços. Entre eles podem ser citados: mudanças nas condiçōes de coagulação e floculação; incremento da taxa de aplicação superficial (TAS) nos tanques de FAD e alterações no sistema de fornecimento de ar. (Schofield, 2000).

Atualmente, um dos grandes limitantes ao aprimoramento de projetos de unidades de flotação reside, sem dúvida nenhuma, no conhecimento dos mecanismos de formação de microbolhas, na distribuição de tamanho e na interação destas com as partículas presentes (fase sólida). Ives (1995) comenta que estas "caixas pretas" podem resultar em projetos insatisfatórios e que a otimização das unidades de FAD, no estado da 
arte atual, passa necessariamente pelo conhecimento das características e das interações entre as partículas (bolhas e flocos), descrita pelo autor como "processos internos".

O tamanho das microbolhas formadas depende da pressão de saturação $\left(\mathrm{P}_{\mathrm{sat}}\right)$, temperatura $(\theta), \mathrm{pH}$, dispositivo de despressurização $\left(\mathrm{D}_{\mathrm{dp}}\right)$, tensão superficial do líquido $(\zeta)$ e razão de recirculação $(\mathrm{p})$. No entanto, a aglutinação das microbolhas após a sua formação também pode interferir na eficiência global do processo. Reali (1991) comenta que o estudo do fenômeno de aglutinação das microbolhas de gás no interior das unidades de FAD é muito importante, pois a obtenção de bolhas de pequenas dimensões é fundamental para o processo. $\mathrm{O}$ mesmo autor ainda coloca que não se tem notícia de estudos específicos sobre a aglutinação de microbolhas no interior de reatores de FAD. A literatura reporta que a faixa usual de tamanho de microbolhas para a FAD é de 10 a $120 \mu \mathrm{m}$ sendo o tamanho médio na faixa de 40 a $60 \mu \mathrm{m}$. De forma simplificada, pode-se afirmar que o fenômeno de aglutinação de microbolhas depende de dois aspectos fundamentais: i) taxa de colisão entre as microbolhas; ii) características da superfície das bolhas e do líquido em torno das mesmas (Reali, 1991).

Em termos gerais, a determinação de tamanho de partículas em tecnologias aplicadas ao saneamento pode ser realizada utilizando-se diferentes princípios de medição. As técnicas mais utilizadas no saneamento são aquelas que empregam o princípio da difração a laser (LALLS), da interferência de campo (através da eletroresistividade Coulter e do bloqueio de luz) e a análise de imagem. Entretanto, o movimento ascensional e a fragilidade da estrutura exigem cuidados adicionais para amostragem das microbolhas.

Rodrigues e Rubio (2003) apresentam alguns métodos de medição de bolhas utilizados na área de mineração e na área ambiental para diferentes tipos de unidades de flotação. A Tabela 1 apresenta outros artigos, complementa as informações apresentadas pelos autores e reapresenta alguns dos métodos utilizados para FAD por se tratar do escopo deste artigo.

Apesar das várias técnicas e equipamentos disponíveis, o alto custo dos equipamentos e suas limitações, principalmente no que se refere à amos- tragem, restringiram a determinação de partículas (floculentas e bolhas de gás) aplicadas às técnicas de tratamento no saneamento. A frágil estrutura das microbolhas presentes em unidades de FAD e a possibilidade de aglutinação das mesmas constituem as principais causas da deformação dos resultados quando métodos intrusivos ou que utilizam extração de amostras são aplicados. Em contraposição, o desenvolvimento e a popularização da tecnologia de câmeras digitais facilitaram seu uso, permitindo uma reflexão sobre seu potencial de utilização na avaliação de microbolhas aplicadas ao processo de clarificação de águas destinadas ao abastecimento.

Portanto, o artigo foca na apresentação do método desenvolvido e aplicado para aquisição, tratamento e obtenção das feiçóes de interesse de microbolhas em movimento, sem extração de amostras e por meio de método não-intrusivo que emprega análise de imagem digital.

O método desenvolvido foi desenvolvido e aplicado em Moruzzi (2005) onde foi possível avaliar, entre outros, a interferência de parâmetros de projeto de unidades de flotação por ar dissolvido (FAD) na distribuição de tamanho de microbolhas (DTMB), bem como o efeito do tamanho dos flocos na eficiência do processo. O ferramental desenvolvido forneceu subsídios importantes para a avaliação da interferência da distribuição de tamanho de partículas em sistemas de separação sólido/líquido por flotação.

A seguir é apresentado e discutido o método proposto para captura de imagens de microbolhas em movimento e, subseqüente tratamento dos arquivos imagens. Posteriormente, no item 3.1 é apresentado o tratamento dos dados e feitas consideraçóes a respeito da precisão do método proposto. Finalmente, no item 3.2 são apresentados os resultados obtidos com a aplicação do método em uma unidade piloto de flotação por ar dissolvido (FAD) e tecidas as consideraçôes finais a respeito do método proposto contemplando generalizações e restrições.

Acredita-se que o método proposto apresenta potencialidade para outras aplicaçôes na área tendo em vista sua simplicidade, baixo custo em relação às demais tecnologias disponíveis no mercado, aplicabilidade às tecnologias de tratamento e adaptabilidade (flexi- bilidade) às condições específicas para cada caso.

\section{MATERIAL E MÉTODOS}

A seguir, serão descritos os procedimentos adotados para a captura e tratamento de imagens das microbolhas em movimento, presentes no processo de separação sólido/líquido por FAD. Inicialmente, apresentaram-se as consideraçôes bem como a descrição metodológica de captura das imagens em movimento. Posteriormente, são apresentados o tratamento e a obtenção das feições de interesse das imagens capturadas, por meio de procedimento metodológico padronizado, e a precisão do método.

\section{Captura das imagens em movimento}

Devido ao movimento ascensional das microbolhas e a fragilidade de sua estrutura, foram desenvolvidos e adaptados procedimentos específicos para a tecnologia de flotação por ar dissolvido (FAD). O desafio foi estabelecer um método para captura das imagens de microbolhas em amostras indeformadas, através da utilização de instalação em escoamento contínuo, combinada com método não-intrusivo de aquisição de imagem. Para tal finalidade, as imagens das microbolhas foram adquiridas em instalação especialmente concebida, denominada Unidade Separadora de Microbolhas (USMB). A Figura 1 apresenta um esquema da instalação piloto objeto da investigação e da unidade denominada USMB.

A USMB possuía dimensões idênticas às da zona de reação da unidade piloto de FAD e foi concebida visando capturar imagens em movimento (fluxo de água em escoamento contínuo), de modo a não interferir nas características originais das microbolhas. O sistema foi construído em vidro para permitir a aquisição externa das imagens, constituindo assim um sistema não-intrusivo. A entrada de água foi feita por meio de Manifold de distribuição, localizado na porção inferior da unidade. Nesta, foi conectado mangueira flexível proveniente da bifurcação do sistema de alimentação da instalação piloto, a jusante do medidor de vazão de entrada. O sistema de recirculação de água saturada foi composto por 3 registros de agulha idênticos aos utilizados na insta- 


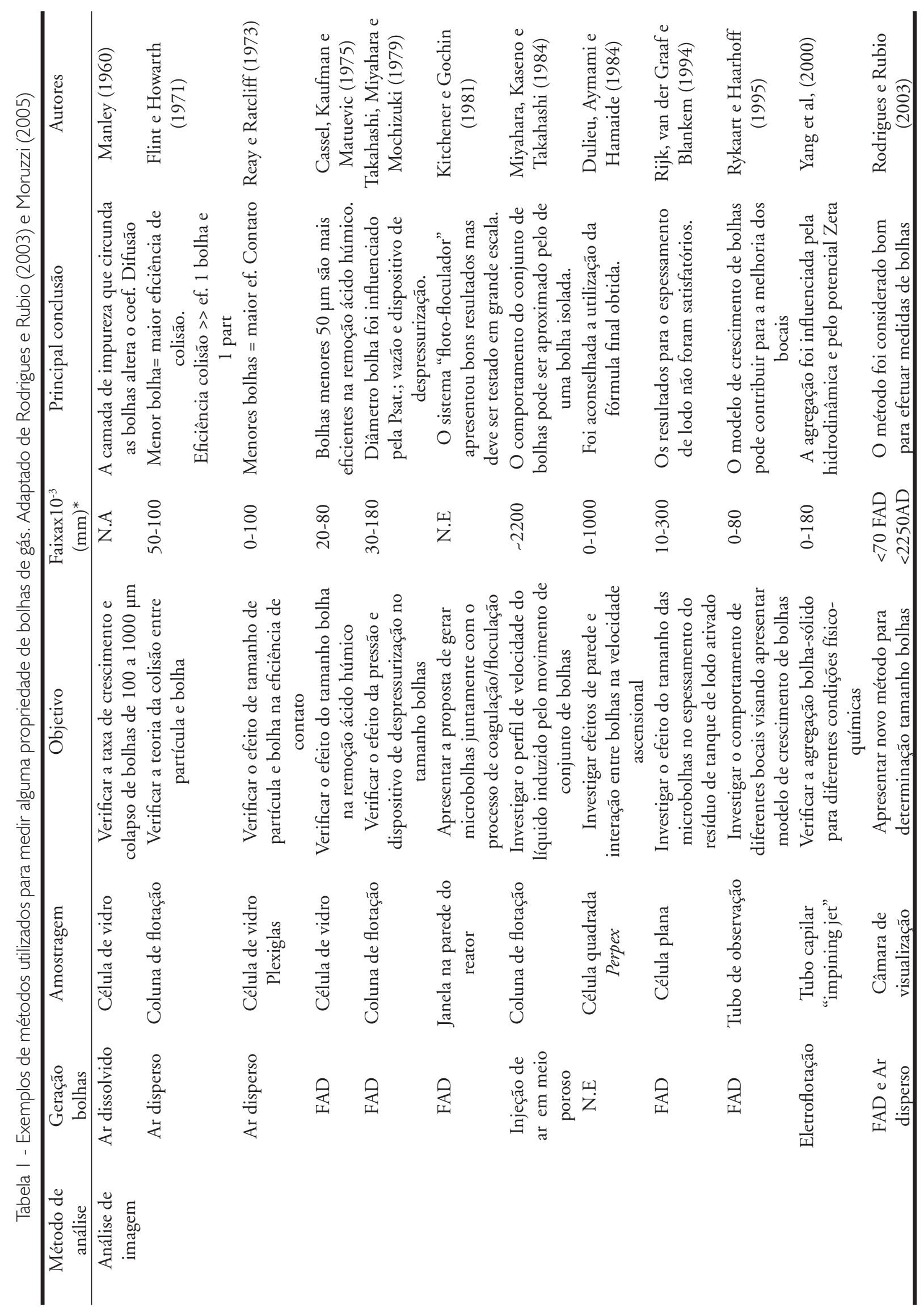


$\mid$

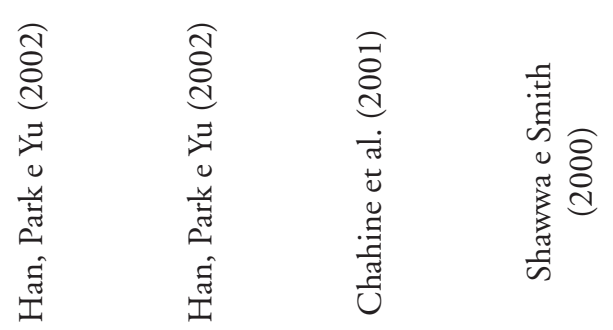

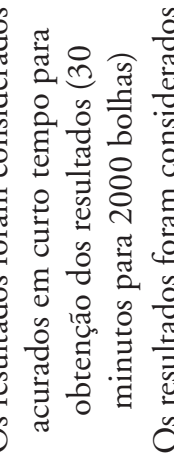

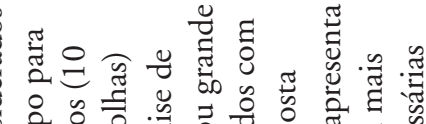

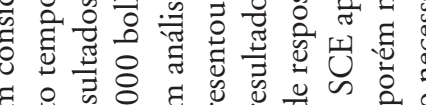

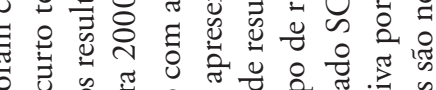

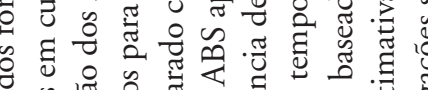

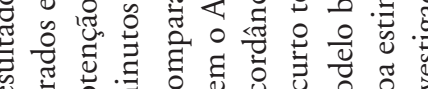

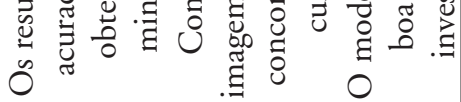

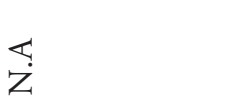

ํํำ

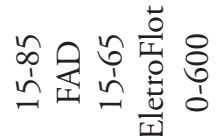

$\stackrel{8}{\circ}$

ชับำ

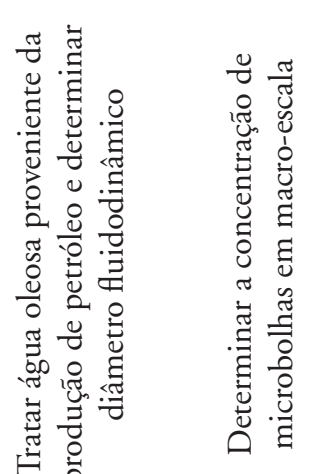

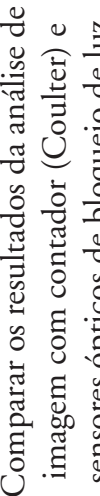

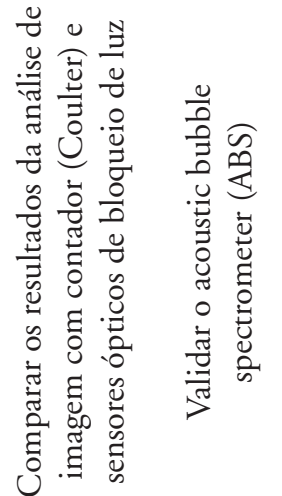

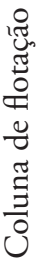

苟

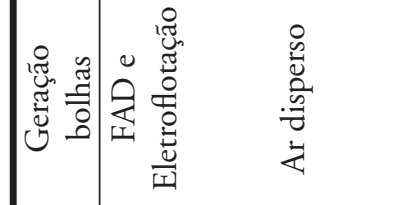

釜

空善

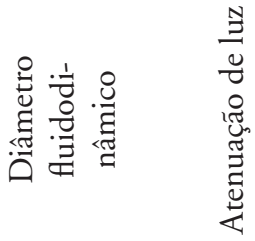

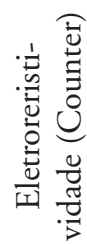
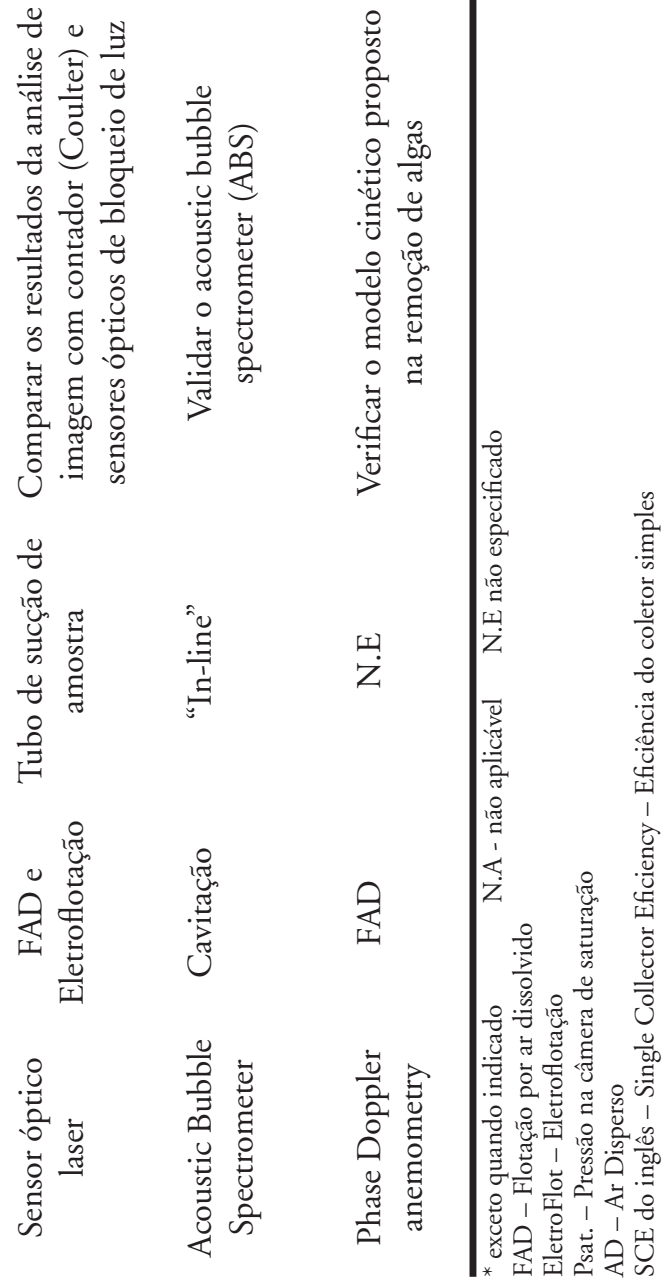
lação piloto. No sistema de registros foi conectada a mangueira flexível proveniente da mesma câmara de saturação utilizada na instalação piloto.

O sistema de saída foi composto por dois Manifold(s) independentes localizados na parte superior da unidade e separados em regióes distintas. A área de coleta e o número de furos de cada Manifold foram calculados para uma coleta uniforme e de tal forma que a soma das áreas dos furos, para cada Manifold, fosse proporcional à área da seção transversal de cada região no ponto de aquisição das imagens. Assim, foi possível separar parte do fluxo de entrada, eliminando o problema proveniente da alta concentração de microbolhas. Durante a operação da USMB, as saídas eram ajustadas na mesma posição, de modo que a amostra fosse coletada proporcionalmente à área de cada seção independente.

A aquisição das imagens foi feita cerca de $20 \mathrm{~cm}$ acima do ponto de despressurização na porção transversal da unidade. A fonte fria de iluminação foi proveniente de um equipamento de $100 \mathrm{~mW}$ de potência que produz um plano de laser de $2 \mathrm{~mm}$ que "corta" a unidade em plano perpendicular à aquisição das imagens. A temperatura foi mantida constante em $22^{\circ} \mathrm{C}$. Devido à ampliação óptica adotada, a área monitorada foi mantida constante em valor determinado. Um fio metálico de 0,160mm foi utilizado como referência na calibração. As imagens digitais foram adquiridas com uma câmera digital "coolpix" modelo Nikon 5400 de 5.1MPixel, com ajuste de foco manual e programada para disparo múltiplo a 1/500, foi acoplada a conjunto de lentes com 60x de ampliação. A análise das imagens adquiridas foi realizada com o software Image Tool versão 3.0 e os procedimentos adotados foram descritos no item 2.2 deste artigo. Para determinação do diâmetro das microbolhas adotou-se o diâmetro estatístico da esfera equivalente de mesma área projetada no plano $\left(\mathrm{D}_{2,0}\right)$. Foram contadas cerca de 11450 microbolhas e o diâmetro médio foi obtido por análise estatística dos dados. Adicionalmente, foram determinados os valores de $\mathrm{D}_{3,2}$ (superfície/volume) também conhecido como $\mathrm{D}_{\text {Sauter }}$ e os resultados comparados. Com isso foram capturadas imagens de microbolhas em movimento no interior da USMB. A Figura 2 apresenta mais detalhes da referida instalação e do arranjo dos periféricos.

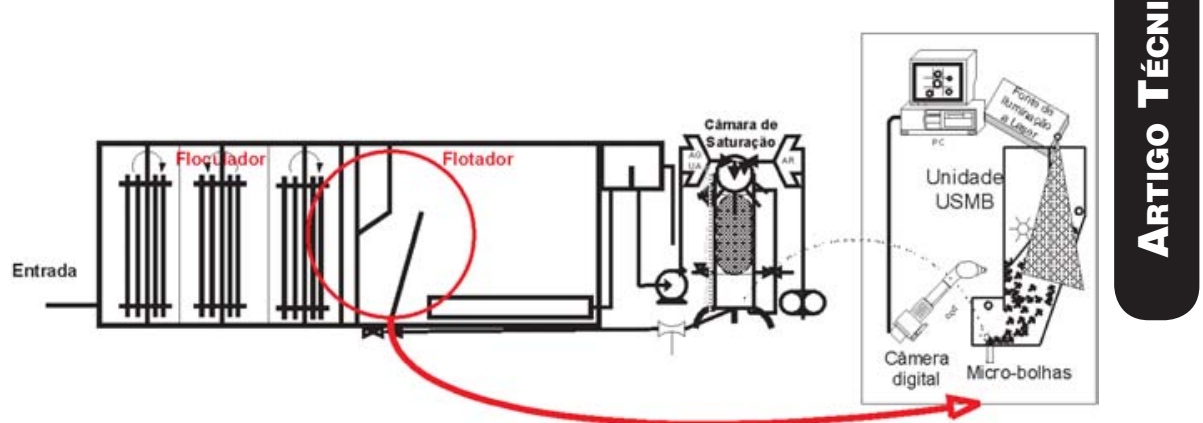

Fonte: Adaptado de Moruzzi (2005).

Figura I - Esquema ilustrativo das instalações em escoamento contínuo utilizadas para a aquisição das imagens visando determinação de tamanho das microbolhas presentes na unidade piloto de FAD

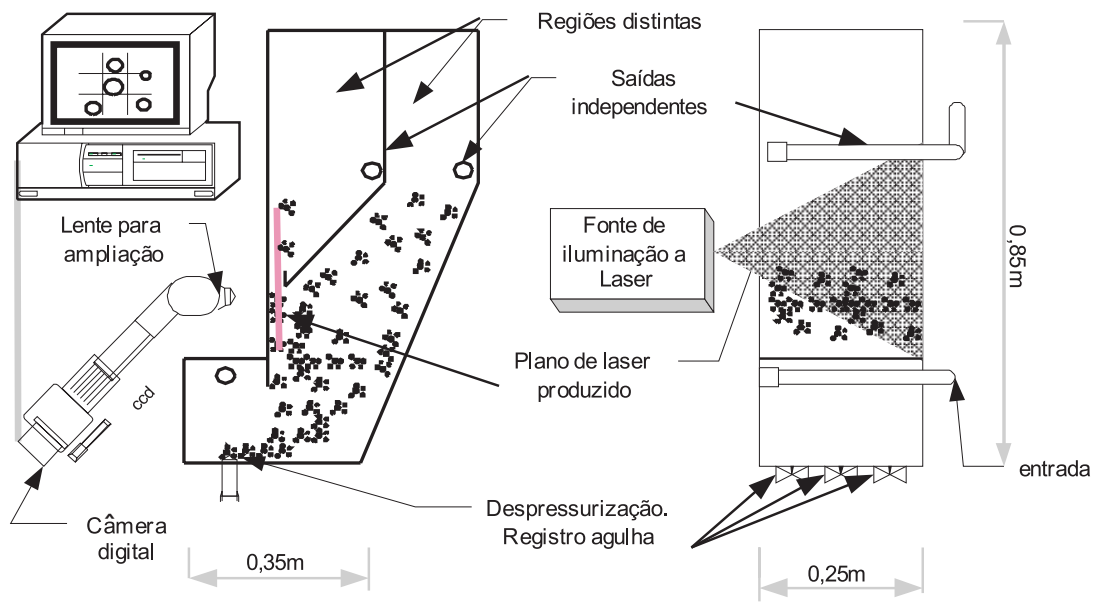

Fonte: Adaptado de Moruzzi (2005)

Figura 2 - Desenho esquemático do arranjo experimental da instalação em escoamento contínuo, denominada unidade separadora de microbolhas (USMB) utilizada para aquisição das imagens e determinação da distribuição de tamanho de microbolhas

O sistema desenvolvido para aquisição de imagens é composto dos seguintes itens:

- unidade para separação/visualização das microbolhas (USMB) em escoamento contínuo;

- microscópio montado em braço mecânico e posicionado na horizontal;

- câmera fotográfica digital com controle manual de foco, velocidade do obturador e função de aquisição múltipla de imagens;

- tripé;

- fio com diâmetro conhecido para calibração;

- iluminação fria constituída de plano de laser.

Após intensiva investigação pôdese propor os seguintes passos para procedimento de captura de imagens das microbolhas:
1- Ligar a entrada de água da unidade através do acionamento da bomba que interliga o reservatório à unidade;

2- Estabelecer o N.A desejado na unidade;

3- Posicionar o fio de referência;

4- Focar o fio utilizando o conjunto de lentes;

5- Montar a câmera no tripé e posicioná-la na ocular do conjunto de lentes, através do adaptador, até visualização de todo campo;

6- Ligar o equipamento de laser e focar o plano de laser no fio de referência, perpendicularmente ao plano de foco;

7- Fixar o foco da máquina no fio de referência (ajuste de foco manual) e fotografá-lo algumas vezes;

8- Retirar o fio de referência sem alterar o foco e o arranjo dos periféricos; 
9- Ligar a câmera de saturação, regular pressão até alcançar estabilidade e abrir registros de distribuição de água saturada;

10 - Regular a máquina para acionar o modo de captura múltipla de imagens na velocidade de abertura de $1 / 500$ e manter o botão de disparo pressionado de modo a capturar as imagens múltiplas;

11- Descarregar os arquivos imagens no PC e repetir procedimento até obter o número de imagens desejadas.

A Figura 3 apresenta um esquema ilustrativo dos passos descritos.

Durante todo o processo de aquisição das imagens não pôde haver nenhuma alteração no arranjo físico da instalação e periféricos. Deviam ser mantidas rigorosamente constantes as condiçôes operacionais da câmara de saturação, vazões (entrada, recirculação, dosadoras de produtos), nível de água, posicionamentos e focagem da câmera e iluminação. Para a obtenção de tamanhos é imprescindível a aquisição da foto de calibragem para cada condição investigada, uma vez que uma pequena variação no posicionamento do sistema óptico pode interferir nos resultados obtidos.

A seguir apresentam-se os procedimentos para obtenção das feições de interesse.

\section{Obtenção das feições de interesse das microbolhas}

O tratamento das imagens foi realizado no programa freeware Image-Tool 3.0®, desenvolvido para a área de medicina. O programa pode ser copiado sem custos a partir do site da The University of Texas Health Science Center at San Antonio e opera perfeitamente na plataforma Windows. A Figura 4 apresenta os passos práticos para download e instalação do software.

\section{$\underline{\text { Calibração }}$}

Primeiramente, deve-se proceder à calibração do sistema através da foto de calibração adquirida antes da obtenção de cada bateria de ensaios. Este procedimento informa o tamanho correspondente a uma determinada distância entre pontos e constitui-se etapa fundamental na determinação da Distribuição de Tamanho de Partículas (DTP) por análise de imagem. Existem 3 passos para a calibração do programa, sendo:

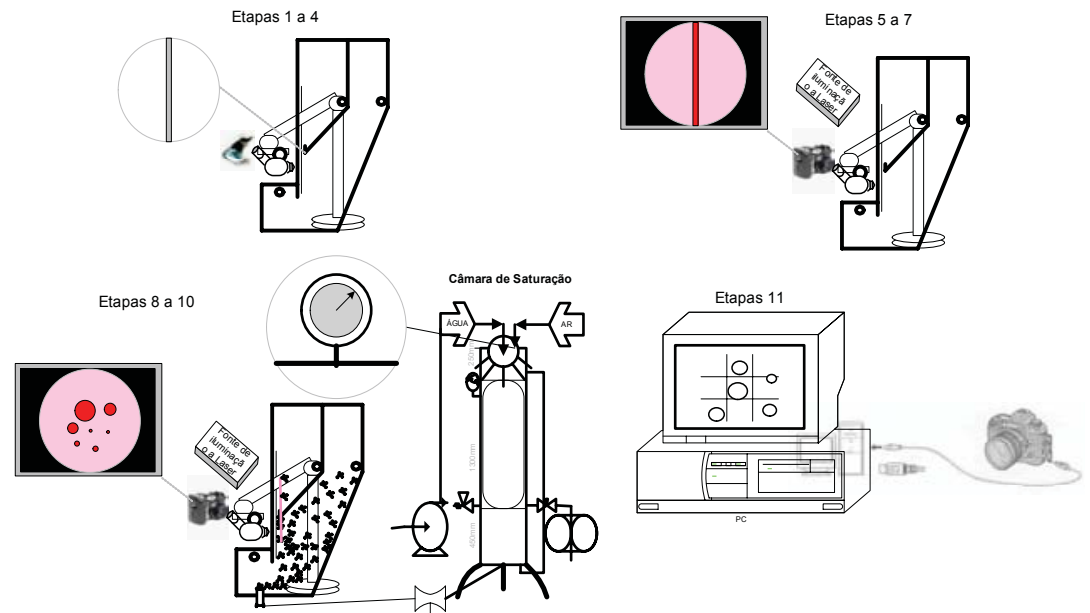

Fonte: Adaptado de Moruzzi (2005).

Figura 3 - Desenho esquemático ilustrativo da seqüência dos passos para aquisição das imagens na unidade separadora de microbolhas (USMB) e determinação da DTMB na unidade piloto de FAD

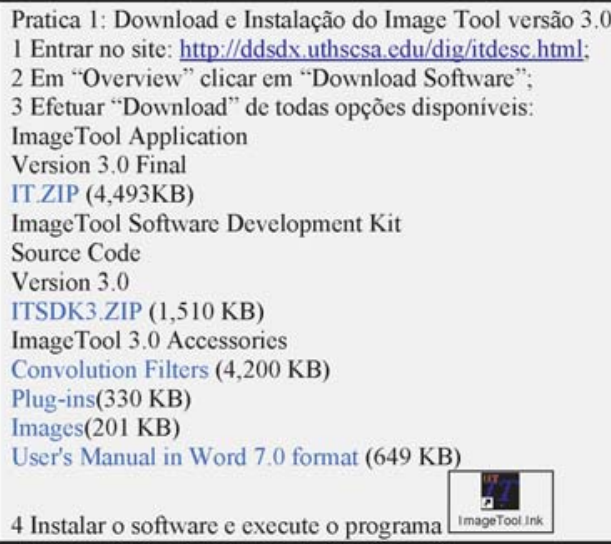

Figura 4 - Prática para Download e Instalação do Image Tool versão 3.0

1 - abertura do arquivo foto;

2- definição manual do limiar de binarização (opcional para a etapa de calibração, pois depende da aquisição da imagem);

3- calibração propriamente dita.

$\mathrm{Na}$ calibração, a binarização da imagem pode não ser necessária quando a imagem for nítida suficiente para delimitação das bordas. Para o programa em questão é importante que tamanho da foto de calibração (resolução) seja o mesmo das imagens adquiridas para o tratamento.

Para a binarização da imagem clicar em Processing no menu e transformar a imagem em escala de cinza utilizando o comando Color-to-grayscale.
Caso a imagem tenha sido adquirida em escala de cinza esta etapa é dispensada. Em seguida no menu Processing escolher a opção threshold no modo manual ou clique no atalho $\square$ na barra de ferramentas. O histograma de freqüência de intensidade é então apresentado. Para imagens em 8 bits a intensidade para cada pixel é dada por $2^{8}$ níveis de cinza que vai de 0 a 255 correspondendo as 256 intensidades do preto ao branco, respectivamente. O limiar de binarização deve ser delimitado manualmente para cada imagem a ser tratada e constitui etapa fundamental no tratamento das imagens.

Posteriormente, procede-se à calibração propriamente dita. No menu, 
clicar em Settings e escolher a opção Calibrate Spatial Measurements. Em seguida, deve-se informar a linha de comprimento conhecido clicando no fio de referência e informar o tamanho e a unidade de medida correspondente.

Em seguida, a calibração deve ser salva. Assim, todas as imagens adquiridas naquelas condições poderão ser medidas através da escolha da calibração correspondente sem a necessidade de repetir a operação. A Figura 5 apresenta os procedimentos práticos para a calibração do programa.

Tratamento das imagens e obtenção dos tamanhos

O tratamento é intimamente determinado pela qualidade das imagens adquiridas e pode ser alterado em função desta etapa. O tratamento das imagens é composto basicamente pelas seguintes etapas:

- Transformação da imagem em escala de cinza (depende da aquisição);

- Binarização manual da imagem (transformação em imagem de 1 bit);

- Segmentação e detecção dos objetos, e;

- Obtenção dos tamanhos.

Caso haja objetos com interseção visível, utilizar a ferramenta Watershed no menu Processing para separá-las. Posteriormente, inicia-se a análise dos objetos. Para tal, clicar em Analysis no menu e escolher a opção Object Analysis e Find Objects.

Como a imagem já foi binarizada em etapas anteriores a opção None (image is already thresholded) deve ser escolhida. A janela de visualização informando o número de objetos identificados aparece. Os objetos são então delineados e o número de cada objeto é apresentado. Depois de identificados, os objetos devem ser analisados. Esta etapa depende da característica da partícula que se pretende obter. Resumidamente, os objetos podem ser analisados através das diferentes propriedades disponíveis no software tais como: diâmetros, áreas, circularidade, perímetro etc. Outra opção consiste em classificar os objetos em faixas de tamanho. Para isso clicar em Analysis no menu, escolher a opção Object Analysis e Classification. $\mathrm{Na}$ janela visual de classificação, a propriedade a ser analisada deve ser selecionada em Attribute. A primeira classe contém objetos com tamanho até o valor esti- pulado. A última classe contém objetos com valores maiores que o estipulado. As demais classes contêm objetos com tamanho entre a classe correspondente e a anterior.

Evidentemente, podem ser criadas rotinas através do script do programa - que pode implicar em significativa economia de tempo no tratamento e obtenção das classes de tamanho dos objetos de cada arquivo imagem. No entanto, o uso desta opção pode incorrer em erros grosseiros na etapa de binarização (transformação em imagem 1bit), podendo comprometer os resultados finais, já que a visualização das imagens é essencial para identificação do limiar de binarização.

A Figura 6 apresenta o roteiro prático para tratamento das imagens e obtenção dos tamanhos.

\section{RESULTADOS E DISCUSSÃO}

\section{Precisão do método}

Existem duas principais fontes de erro no método utilizado: i) erro referente à amostragem; ii) erro inerente ao método de tratamento e análise das imagens.

O erro na amostragem depende do número de amostras analisadas frente à população total e do dispositivo de coleta de microbolhas. Este último é inerente ao método de coleta utilizado e não pode ser estimado precisamente. Neste trabalho foi utilizado a USMB de modo que as imagens foram adquiridas no interior da zona de reação sem a necessidade de se realizar a coleta de amostras para posterior análise. Assim

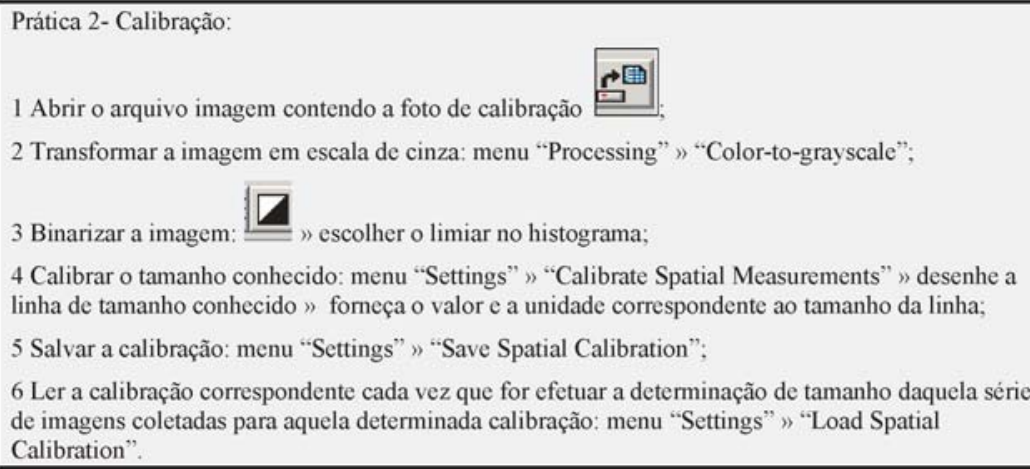

Fonte: Adaptado de Moruzzi (2005)

Figura 5 - Prática para calibração do programa
Prática 3 - Tratamento das imagens e obtenção dos tamanhos

1 Abrir o arquivo imagem contendo os objetos a serem analisados

2 Transformar a imagem em escala de cinza: menu "Processing" " "Color-to-grayscale";

3 Binarizar a imagem " escolher o limiar no histograma

4 Separar os objetos que porventura estejam unidos: menu "Processing" " "IP*EDM" " "Watershed";

5 Delimitar a área de cada objeto: menu "Analysis" " "Object Analysis" ” "Find Objects" " opção

"None (image is already thresholded)";

6 Classificar os objetos identificados em faixas de tamanho: menu "Analysis" " "Object Analysis" ” "Classification";

7 Escolher a faixa de tamanho pertinente de acordo com a propriedade em que deseja analisar ou leia classe previamente estipulada;

8 Efetuar os passos para cada arquivo imagem a ser analisado:

9 Salvar os dados da janela visual "Results" e copiar os dados para planilha eletrônica para consecução dos gráficos: menu "File" " "Save Results As"

Fonte: Adaptado de Moruzzi (2005).

Figura 6 - Prática para tratamento das imagens e obtenção dos tamanhos 
eliminou-se o erro referente à coleta de amostras.

Estima-se cerca de $100000 \mathrm{mi}$ crobolhas $/ \mathrm{ml}$ para $0,075 \mathrm{ml} \mathrm{ar} / \mathrm{ml}$ água e microbolhas de diâmetro médio de $50 \mu \mathrm{m}$. Para as razóes de recirculação estudadas ( $p=0,1$ e 0,05$)$ o número de microbolhas liberadas no sistema foi da ordem de $5 \times 10^{7}$ e $10^{8}$ microbolhas/minuto. Assim, para o cálculo do número de microbolhas foi considerada uma população infinita. No entanto, no momento da aquisição das imagens não foi possível controlar o número de microbolhas adquiridas. O número de microbolhas foi determinado somente após a aquisição e análise dos dados. Sendo assim, foram adquiridas o máximo de imagens possível e o erro foi calculado para cada condição investigada. O cálculo do erro amostral é matéria conhecida e, apesar de imprescindível para análise de resultados experimentais, não foi apresentado por não se tratar do escopo do presente artigo.

O segundo erro refere-se ao método de análise e tratamento de imagem utilizado, mais precisamente, na etapa de binarização manual da imagem.

As imagens foram adquiridas de tal modo que não foi possível identificar os objetos antes do tratamento (in-loco), pois a velocidade de captura para congelar as microbolhas em movimento foi de $1 / 500$ e a iluminação foi propositalmente insuficiente para a visualização dos objetos antes do tratamento. Desta forma, na etapa de binarização manual das imagens, foi possível separar mais precisamente as microbolhas que passaram pelo foco, pois estas apareciam com maior nível de brilho sendo mais facilmente detectadas.

O diâmetro estatístico utilizado foi o da esfera de mesma área equivalente. A área equivalente de cada objeto identificado (micro-bolha) foi definida em função da área dos pixel(s) (menor elemento unitário que compóem a imagem) contidas em cada micro-bolha (Figura 7). O tamanho de cada pixel depende da resolução adotada e do grau de aumento utilizados na captura da imagem. Quanto maior a resolução e o grau de aumento menor o tamanho do pixel e, assim maior a precisão na definição das bordas do objeto. Entretanto, quanto maior o grau de ampliação, menor a área de captura, e assim, menos bolhas são capturadas por arquivo imagem e mais arquivos devem ser tratados para uma mesma precisão estatística. Da mesma forma, quanto maior a resolução, mais informaçóes a imagem contém e, portanto, mais difícil de trabalhar no software.

A escolha das condições adequadas de captura depende do tamanho médio dos objetos que serão analisados, da capacidade de processamento do sistema de aquisição e do erro requerido.

Para a aquisição das imagens apresentadas neste artigo, foi utilizada ampliação óptica de 60x para resolução da imagem de 480x640 supondo inicialmente diâmetro médio de microbolhas de $0,050 \mathrm{~mm}$. Assim, o tamanho do pixel foi de aproximadamente $0,005 \mathrm{~mm}$.

O erro no tratamento foi determinado em função da área dos pixel(s) que continham a imagem para cada tamanho médio das microbolhas nas faixas investigadas. Para cada faixa investigada, a área pode ser sub ou superestimada (Figura 7) dependendo do limiar de binarização adotado. Assim, o erro foi calculado em função da área de pixel(s) (para mais ou para menos) em relação à área do objeto.

A Tabela 2 apresenta o erro do método para cada faixa de tamanho de microbolhas. Evidentemente, quanto menor a micro-bolha, maior o erro na determinação de sua borda (para um tamanho fixo de pixel). Para microbolhas de $0,050 \mathrm{~mm}$ o erro inerente ao tratamento foi menor que $0,50 \%$ $(\sim 0,30 \%)$.

\section{Estudo de caso}

Utilizando o método proposto, foi verificada a variação da distribuição de microbolhas na ausência e presença de concentração fixa do cátion metálico $\mathrm{Al}^{+3}$, determinada em ensaios de coagulação com a água de estudo em escala de laboratório. A Figura 8 apresenta uma imagem típica das microbolhas adquiridas após binarização da imagem original, conforme procedimento apresentado. A Figura 9 apresenta como exemplo, uma variação típica da variação da distribuição de tamanho de microbolhas (DTMB) na ausência e presença do cátion $\mathrm{Al}^{+3}$. Para este estudo de caso, foram analisadas aproximadamente 11540 microbolhas garantindo amostras com $95 \%$ de intervalo de confiança para erro amostral de $1 \%$.

Verificou-se que houve um discreto aumento no número de bolhas com diâmetros menores que $50 \mu \mathrm{m}\left(\mathrm{d}_{50}\right)$ quando não foi aplicado coagulante (Figura 9 a-b). O diâmetro médio $\left(\mathrm{D}_{\text {médio }}\right)$ foi praticamente o mesmo na presença ou ausência de coagulante (de $27 \mu \mathrm{m}$, sem $\mathrm{Al}^{+3}$ para $29 \mu \mathrm{m}, \mathrm{com} \mathrm{Al} \mathrm{Al}^{+3}$ ) pois a variação em número das microbolhas foi pouco sensível devido ao elevado percentual do $d_{50}$ (Figura 9-b). No entanto, ao se considerarmos o diâmetro estatístico $D_{\text {Sauter }}\left(D_{3,2}\right)$, notou-se uma variação de 40 para $48 \mu \mathrm{m}$ quando o coagulante $\left(\mathrm{Al}^{+3}\right)$ foi aplicado para as mesmas condições de mistura e tempo de detenção, indicando que o fenômeno

Tabela 2 - Erro no tratamento das imagens (binarização) para algumas faixas de tamanho de microbolhas. Tamanho pixel de $5 \mu \mathrm{m}$

\begin{tabular}{|c|c|c|c|c|}
\hline $\begin{array}{c}\text { Diâmetro } \\
\text { micro-bolha }(\mu \mathrm{m})\end{array}$ & $\begin{array}{l}\text { Área real* } \\
\quad\left(\mu \mathrm{m}^{2}\right)\end{array}$ & $\begin{array}{c}\text { Diâmetro equivalente com base } \\
\text { na área subestimada }\left(\mu \mathrm{m}^{2}\right)\end{array}$ & $\begin{array}{c}\text { Diâmetro equivalente com base } \\
\text { na área superestimada }\left(\mu \mathrm{m}^{2}\right)\end{array}$ & $\begin{array}{c}\text { Faixa de Erro } \\
(\%)\end{array}$ \\
\hline 10 & 78 & 9 & 11 & $2,20-12,80$ \\
\hline 20 & 314 & 19 & 20 & $1,70-2,30$ \\
\hline 50 & 1963 & 49 & 50 & $0,28-0,30$ \\
\hline 100 & 7853 & 99 & 100 & $0,03-0,13$ \\
\hline 110 & 9503 & 109 & 110 & $0,02-0,12$ \\
\hline 120 & 11309 & 119 & 120 & $0,04-0,06$ \\
\hline
\end{tabular}




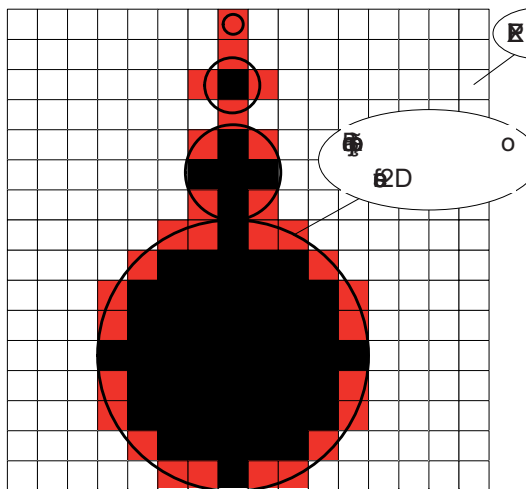

Fonte: Adaptado de Moruzzi (2005).

Figura 7 - Exemplo hipotético da área dos pixel(s) em função do tamanho médio das microbolhas

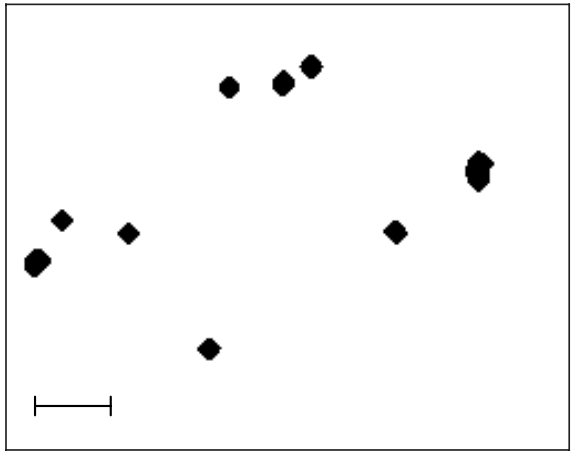

Fonte: Adaptado de Moruzzi (2005).

Figura 8 - Exemplo típico de imagem de microbolhas adquirida digitalmente após binarização utilizando freeware Image Tool 3.0. $P_{\text {sat }} 450 \pm 10 \mathrm{KPa}, \mathrm{T}_{2}^{\circ} \mathrm{C}$

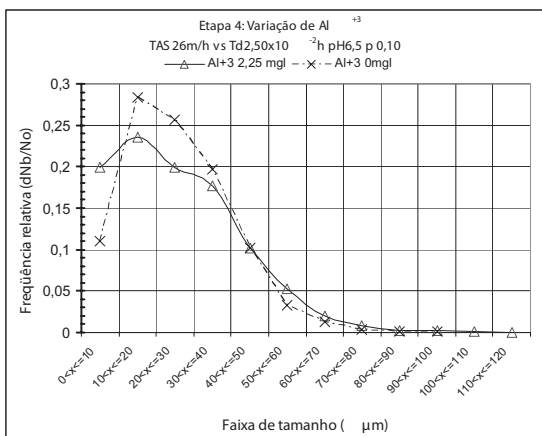

a)

Figura 9 - Freqüência relativa(a), acumulada(b) em número de microbolhas e (c) análise amostral estatística dos resultados com desvio padrão. Resultados dos ensaios de DTMB obtidos através método não intrusivo que emprega análise de imagem na USMB na presença e ausência de $\mathrm{Al}^{+3}$ Fixos: TAS e Td, $P_{\text {sat }} 450 \pm I 0 K \mathrm{~Pa}, \theta 22 \pm I^{\circ} \mathrm{C}, \mathrm{D}_{\mathrm{dp}}$ registros de agulha $I / 8^{\prime \prime}, \mathrm{pH}, \mathrm{p}$ da aglutinação foi ligeiramente mais acentuado na presença do cátion $\mathrm{Al}^{+3}$. Esta observação encontra respaldo nos resultados obtidos por Cassel, Kaufman e Matuevic (1975) onde também foi observado um discreto incremento no diâmetro médio das microbolhas com a adição de $\mathrm{Al}^{+3}$. Entretanto, esta comparação pode não ser representativa uma vez que o tamanho das microbolhas sofre interferência do método de análise bem como dos dispositivos de geração e despressurização (câmara de saturação, bocais ou registros de agulha).

Através dos resultados do estudo de caso apresentado, procurou-se ressaltar a importância da análise da distribuição de tamanho de microbolhas em sistemas de FAD. O método proposto constitui uma importante ferramenta para compreensão dos fenômenos envolvidos em processos de tratamento, pois conforme preconizado Tchobanoglous (1995), os parâmetros não-específicos comumente empregados em sistemas de tratamento não são sensíveis a este tipo de análise.

No item seguinte, uma avaliação crítica do método proposto para determinação das características das partículas utilizando a aquisição e tratamento da imagem digital.

\section{Avaliação crítica a respeito do método proposto}

A avaliação do método proposto e de sua aplicação, através dos resultados do estudo de caso para a FAD, permite vislumbrar a respeito da confiabilidade, aplicação, flexibilidade e importância deste ferramental na avaliação de sistemas de tratamento de água para abastecimento.

Apesar de existirem métodos de determinação de tamanho de partículas propostos pelo Standard Methods for the Examination of Water and
Wastewater desde sua $18^{a}$ edição, estes consideram apenas dispositivos eletrônicos de medida (sensor de zona elétrica, bloqueio de luz e espalhamento de luz), equipamentos esses de custo ainda bastante elevado. Ademais, deve-se atentar para o sistema de coleta de amostras.

O método desenvolvido para determinação de tamanho de microbolhas foi concebido segundo um procedimento dedicado e único. Muitos de seus componentes foram adaptados de equipamentos existentes de acordo com as necessidades que se impunham e dessa forma foram totalmente originais. Não são originais os dispositivos ópticos e os demais periféricos que compóem o sistema, no entanto, são originais as formas como estes componentes foram adaptados e conjugados, o que permite que o sistema seja flexível para as diferentes aplicações.

Não é comum, em análise de partículas, o emprego de técnicas in-loco 
sem a extração de amostras. As aplicações usuais, nos mais diferentes princípios de medição, referem-se à análise das partículas a partir da coleta de amostras no interior dos reatores $e$ a posterior análise destas amostras em células de pequenas dimensôes, podendo ocasionar em grandes distorções dos resultados. A necessidade de implantar um sistema de aquisição sem a extração de amostra ocasionou um esforço adicional no desenvolvimento do método, culminando na unidade denominada USMB, sem mencionar os aspectos inerentes à obtenção de imagens em movimento.

A instalação dos dispositivos e periféricos é um fator importante que merece cuidado especial. As medidas tomadas pelo método de aquisição de imagens de microbolhas são micrométricas e alguns detalhes de instalação tais como: alinhamento, posicionamento, manutenção do foco e calibração são essenciais para uma aquisição e registro adequados.

A desvantagem do método, identificada como a mesma em todos os métodos que empregam a análise de imagens, refere-se ao tempo necessário para a obtenção dos resultados. Para o estudo de caso apresentado, foi despendido cerca de 5 minutos no tratamento e obtenção dos tamanhos para cada arquivo imagem. $\mathrm{O}$ grande número de arquivos imagem (cerca de 2000), necessários para a amostragem com elevada representatividade, fez com que o tempo total fosse bastante elevado. Isso porque as imagens devem ser tratadas individualmente além do que, os resultados obtidos para cada um destes arquivos devem ser processados em planilha eletrônica e tratados estatisticamente. Isso faz com que a principal limitação do método seja o tempo de resposta. Evidentemente, o sistema deve contar com um contínuo aprimoramento permitindo a adoção de procedimentos que agilizem o processo de aquisição e tratamento das imagens, contudo o número de amostras necessárias para uma precisão estatística adequada, bem como a necessidade da análise visual para determinação do limiar de binarização, serão alguns dos limitantes. Todavia, acredita-se que rotinas de análise podem permitir a diminuição expressiva do número de amostras analisadas bem como a diminuição do tempo despendido no processo de tratamento.
O tema é complexo e, certamente, não se esgota no presente artigo. No entanto, considera-se que este constituiu um importante passo na direção do aprimoramento das análises para a obtenção de sistemas de tratamento mais econômicos e eficientes. Acredita-se que o método pode ser adaptado para determinação da distribuição de tamanho de flocos bem como para sua análise morfológica.

Assim, o método proposto vai ao encontro dos novos desafios da área de saneamento, mais especificamente na área de tratamento de águas para abastecimento.

A aplicação do método em unidades em escala real depende de diversos fatores relacionados às características particulares de cada sistema. No entanto, dada a flexibilidade do sistema desenvolvido, acredita-se que com adaptaçôes adequadas nas instalações isso seja possível.

\section{CONCLUSÃO}

Verificou-se que a análise de imagem para a distribuição de partículas constituiu uma alternativa viável, para determinação da distribuição de tamanho de microbolhas, do ponto de vista técnico tendo como principais vantagens a simplicidade, a flexibilidade, a possibilidade de coleta de amostras indeformadas e o baixo custo. Entretanto, o tempo gasto para a obtenção dos resultados constitui a principal desvantagem do método apresentado. Com o método proposto foram resolvidos os problemas de deformação dos resultados decorrente da extração de amostras e da alta concentração de microbolhas (através da concepção da USMB). Os resultados apontaram para valores de diâmetros coerentes com os resultados apresentados na literatura, entretanto deve-se fazer uma ressalva a respeito dos métodos utilizados e dos dispositivos de geração e despressurização de microbolhas uma vez que: os métodos com extração de amostra e a característica dos dispositivos de geração e despressurização interferem diretamente na DTMB.

Acredita-se que a incorporação de tais rotinas de análise pode contribuir na evolução do método bem como na compreensão teórica dos fenômenos e no aperfeiçoamento prático de projetos e de operação de sistemas de FAD aplicados ao tratamento de água para abastecimento.

\section{AGRADECIMENTOS}

Os autores agradecem à FAPESP pelo financiamento da pesquisa.

\section{REFERÊNCIAS}

CASSELL, E.A.; KAUFMAN, K.M.; MATIJEVIC, E. The effects of bubble size on microflotation. Wat. Res., v.9, p. 1017-1024, 1975.

CHAHINE, G.L.et al. Validation of bubble distribution measurements of the ABS Acoustic Bubble Spectrometer ${ }^{\oplus}$ with high Speed Video Photography. Disponível em: <http://www.dynaflow-inc. com/Publication_DW/pdf_documents/ sessionA7.004.pdf>. Acesso em: 24 mar. 2001.

EMETS, B.G. Determination of the average size and concentration of air bubbles in water by nuclear magnetic resonance. Tech. Phys. Lett., v. 23, n. 7 , p. 513-514, Jul. 1997.

FLINT, L.R.; HOWARTH, W.J. The collision efficiency of small particles with spherical air bubbles. Chem. Eng. Sci., v. 26, p. 1155-1168, 1971.

HAN, M.Y.; PARK, Y.H.; YU, T.J. Development of a new method of measuring bubble size. Wat. Suppl., v. 2, n. 2, p.77-83, 2002.

IVES, J. K. The inside story of water-treatment processes. Journal of Environmental Engineering, ASCE. v. 121, n. 12, p. 846-849, 1995.

KITCHENER, J.A.; GOCHIN, R.J. The mechanism of dissolved air flotation for potable water: basic analysis and a proposal. Wat. Res., v. 15, p. 585-590, 1981.

LAWLER, D. F. Particle size distribution in treatment processes: theory and practice. Wat. Sci. Tech., v. 36, n. 04, p. 15-23, 1997.

LEPPINEN, D.M.; DALZIEL, S.B.; LINDEN, P.F. Modelling the global efficiency of dissolved air flotation. IWA Publishing. Wat. Sci. Tech., v. 43, n. 8, p.159-166, 2001

MANLEY, D.M.J.P. Change of size of air bubbles in water containing a small dissolved air content. Brit. J. Appl. Phys., v. 11, p. 38-42, Jan. 1960.

MELO, M.V.; PEREIRA JR; MASSARANI, G. Tratamento de águas oleosas por flotação. In: $28^{\circ}$ ENCONTRO SOBRE ESCOAMENTO EM MEIOS POROSOS (ENEMP), Anais... Teresópolis, RJ: UFRJ, 2000.

MIYAHARA, T.; KASENO, S.; TAKAHASHI, T. Studies on chains of bubbles rising through quiescente liquid. Can. J. Chem. Eng., v. 62, p.186-193, Apr. 1984.

MORUZZI, R. B. Avaliação da Influência da Distribuição de Tamanho de Partículas e do Binômio Velocidade/Tempo de Detenção na Zona de Reação no Desempenho da Flotação com Utilização de Sonda Ultrasônica e Técnica de Análise por Imagem. Tese de doutorado apresentada à Escola de Engenharia de São Carlos, Universidade de São Paulo. São Carlos: EESC - USP. 240p, 2005.

REALI, M. A. P. Concep̧̧ão e avaliação de um sistema compacto para tratamento de águas de abastecimento utilizando o processo de flotaçāo por ar dissolvido e filtrą̧ão com taxa declinante. $373 \mathrm{f}$. Tese (Doutorado)Escola de Engenharia de São Carlos, Universidade de São Paulo, São Carlos, 2 v. 1991. 
REAY, D.; RATCLIFF, G.A. Removal of fine particles from water by dispersed air flotation: effects of bubble size and particle size on collection efficiency. Can. J. Chem. Eng., v.51, p. 178-185, April 1973.

RIJK, S.E.; et al. Bubble size in flotation thickening. Wat. Res., v.28, n. 2, p. 465-473, 1994.

RODRIGUES, R. T.; RUBIO, J. New basis measuring the size distribution of bubbles. Minerals Engineering, v. 16, p. 757-765, 2003.

RYKAART, E.M.; HAARHOFF, J. Behaviour of air injection nozzels in dissolved air flotation. Wat. Sci. Tech., v. 31, n. 3-4, p. 25-35, 1995.

SANTOS, H.R.; et al. Aplicabilidade das técnicas de determinação de tamanho de partículas em sistemas de tratamento de água e esgoto sanitário. Engenharia Sanitária e Ambiental, v.9, n.4, p. $291-300-$ out $/$ dez 2004

SCHOFIELD, T. Dissolved air flotation in drinking water production. Wat. Sci. Tech., v.43, n.8, p.9-18, 2000.

SHAWWA, A.R.; SMITH, D.W. Dissolved air flotation model for drinking water treatment. Can. J. Civ. Eng., v. 27, p. 373-382, 2000.

TAKAHASHI, T.; MIYAHARA, T.; MOCHIZUKI, H. Fundamental study of bubble formation in disolved air pressure flotation. J. Chem. Eng. Japan, v. 12 , n. 4 , p. $275-280,1979$

TCHOBAnOglous, G. Particle size characterization: the next frontier. Journal of Environmental Engineering, ASCE. v. 121, n. 12, p. 844-845, 1995.

YANG, C.; et al. A visualizing method for study of micron bubble attachment onto a solid under varying physicochemical conditions. Ind. Eng. Chem. Res., v. 39, p. 4949-4955, 2000.

Endereço para correspondência:

Rodrigo Braga Moruzzi

Departamento de Planejamento Territorial e Geoprocessamento Instituto de Geociências e

Ciências Exatas

(UNESP - Campus de Rio Claro)

Rua I0, n० 2527 - Santana

2002 I-060 Rio Claro - SP - Brasil

Tel.: (I9) 3526-24 I I ramal 2 I4

E-mail: rmoruzzi@rc.unesp.br 